\title{
The Role of Gut Microbiota and Genetic Susceptibility in the Pathogenesis of Pancreatitis
}

\author{
Fumin $\mathrm{Xu}^{1}$, Chunmei Yang ${ }^{1}$, Mingcheng Tang ${ }^{1}$, Ming Wang ${ }^{1}$, Zhenhao Cheng ${ }^{1}$, Dongfeng Chen ${ }^{1}$, Xiao Chen ${ }^{2}$, and \\ Kaijun Liu ${ }^{1}$ \\ Departments of ${ }^{1}$ Gastroenterology and ${ }^{2}$ Nuclear Medicine, Daping Hospital, Army Medical University, Chongqing, China
}

Article Info
Received August 9, 2021
Revised September 6, 2021
Accepted September 17, 2021
Published online December 16, 2021
Corresponding Author
Kaijun Liu
ORCID https://orcid.org/0000-0003-0027-9278
E-mail kliu_tmmu@126.com
Xiao Chen
ORCID https://orcid.org/0000-0002-8647-0614
E-mail xiaochen229@foxmail.com

Fumin Xu and Chunmei Yang contributed equally to this work as first authors.

\begin{abstract}
Pancreatitis is one of the most common inflammatory diseases of the pancreas caused by autodigestion induced by excessive premature protease activation. However, recognition of novel pathophysiological mechanisms remains a still challenge. Both genetic and environmental factors contribute to the pathogenesis of pancreatitis, and the gut microbiota is a potential source of an environmental effect. In recent years, several new frontiers in gut microbiota and genetic risk assessment research have emerged and improved the understanding of the disease. These investigations showed that the disease progression of pancreatitis could be regulated by the gut microbiome, either through a translocation influence or in a host immune response manner. Meanwhile, the onset of the disease is also associated with the heritage of a pathogenic mutation, and the disease progression could be modified by genetic risk factors. In this review, we focused on the recent advances in the role of gut microbiota in the pathogenesis of pancreatitis, and the genetic susceptibility in pancreatitis. (Gut Liver 2022;16:686-696)
\end{abstract}

Key Words: Pancreatitis; Gut microbiota; Genetic susceptibility; Pathogenesis

\section{INTRODUCTION}

Pancreatitis, one of the most common gastrointestinal diseases, is the main cause for hospital admission, and the incidence of pancreatitis is increasing worldwide, which is associated with the elevated socioeconomic burden. ${ }^{1}$ The annual incidence of acute pancreatitis (AP) is approximately 34 per 100,000 in developed countries and it keeps a continuous growth worldwide. ${ }^{2} \mathrm{AP}$ is usually caused by structural obstruction of the biliary tract, alcohol consumption, endoscopic retrograde cholangiopancreatography and drugs, which ultimately lead to acinar cell death, inducing local and systemic inflammation. ${ }^{3-5}$ Chronic pancreatitis (CP) often occurs in patients with recurrent pancreatic injury or prolonged AP. Despite many advances have been made in respects of the pathophysiology of pancreatitis, there are still no medication available to treat or prevent AP at present. ${ }^{6}$ Additionally, in many cases, individuals who even have attacks of alcoholism and gallstones do not suffer from $\mathrm{AP}^{7,8}$ This inclined us to further explore the underlying mechanism of pancreatitis.

The pathogenesis of disease is generally related with genetic and environmental factors, while human gut microbiome is recognized as a potential source of environmental effect on illness. ${ }^{9}$ Recent studies regarding the role of gut microbiome in the pathophysiology of the pancreas are increasing, during which immune regulation and interplay between host microbiomes and the pancreas attract much attention. ${ }^{10-14}$ These studies have initiated new insight into pancreatic diseases from the perspective of the gastrointestinal microbiota. On the other hand, it has long been suspected that genetic susceptibility factors conduce to the pathogenesis of the disease, since only a small proportion of alcoholics finally develop $\mathrm{CP} .{ }^{15}$ Various groups of genetic mutations, such as the serine peptidase inhibitor Kazal type 1 (SPINK1), anionic trypsinogen serine protease 2 (PRSS2), cationic trypsinogen serine protease 1 (PRSS1), cystic fibrosis transmembrane conductance regulator 
(CFTR) genes and so on, were observed in various types of pancreatitis. ${ }^{16,17}$ These specific genetic mutations instruct us to uncover the underlying mechanism of pancreatitis on a genetic and cellular level.

In this review, we summarize recent advances in research of gut microbiota and genetics related to pancreatitis, and analyze the role of the gut microbiota and genetic susceptibility in the pathogenesis of pancreatitis. Additionally, we discuss the relationship between gut microbiota and genetic susceptibility in patients with pancreatitis and are attempting to speculate the pathogenesis of pancreatitis from a novel perspective.

\section{THE ROLE OF GUT MICROBIOTA IN} PANCREATITIS

\section{Acute pancreatitis}

During the course of AP, microcirculatory injury and hypovolemia would emerge, ${ }^{18}$ which could cause intestinal mucosal ischemia and subsequent reperfusion injury, leading to dysfunction of intestinal barrier and gut microbiota translocation. Current investigations have shown heterogeneity in intestinal microbial composition between pancreatitis patients and healthy controls. Zhang et al. ${ }^{19}$ made use of high-throughput $16 \mathrm{~S}$ rRNA gene amplicon sequencing to detect the gut microbiome of $45 \mathrm{AP}$ patients and 44 healthy individuals, and analyzed the differences between the two groups. The results showed that the composition of intestinal flora in AP patients was remarkably changed, and the diversity of their phyla was significantly reduced. Moreover, samples from AP patients had a higher abundance of Proteobacteria and Bacteroidetes, while the abundance of Firmicutes and Actinobacteria was relatively lower when compared with those from healthy individuals (Table 1). Although the imbalance of gut microbiota of AP patients is causative or reactive is still unclear, it is speculated that this may be the inevitable process of the onset and development of AP. Li et al..$^{20}$ detected DNA of Escherichia coli, Shigella and other bacteria in the blood of AP patients, further confirming that intestinal opportunistic bacteria can enter the blood circulation of AP patients through the damaged intestinal barrier, thus aggravating the progression of the disease and the occurrence of infectious complications (Table 1).

When it comes to the evaluation of severity and prognosis of AP, the imbalance of bacterial composition and altered gut microbiota diversity are two overlooked factors and have been gradually emphasized by researchers in recent years. Yu et al. ${ }^{21}$ found that Bacteroides, Escherichia and Shigella, and Enterococcus were dominant intestinal bacterial community in mild, moderately severe, and severe AP, respectively. Moreover, they investigated the relationship between the alterations of gut microbiota and prognosis in hypertriglyceridemia-associated acute pancreatitis (HTGAP) patients in follow-up studies, which showed that HTGAP group had worse prognosis (higher proportion of organ failure and longer hospital stay) and poorer microbial diversity when compared to AP patients with other etiologies (Table 1). ${ }^{22}$ Similarly, Zhu et al. ${ }^{23}$ also reported that the severity of AP is associated with gut microbiota dysbiosis in both human and animal models. These studies suggest the role of gut microbiota might play in evaluating patients and as potential target for treatment.

Though the efficacy of probiotics, such as Bifidobacterium and Lactobacillus, in treatment of severe acute pancreatitis (SAP) is controversial according to earlier clinical trials, ${ }^{24-27}$ updated studies hold optimistic view in application of probiotics in therapy with SAP. One recent metaanalysis concluded that probiotics have beneficial effects on decreasing duration of hospital stay and reducing risk of organ failure in patients with SAP. ${ }^{28}$ In animal models, Lei et al. ${ }^{29}$ found that Parabacteroides could alleviate AP in heparanase-transgenic mice by reducing neutrophil infiltration. Their mechanism research indicated that acetate derived from this gut microbiota genera reduced neutrophils in blood and resulted in less neutrophil infiltration in the pancreas, and thereby enhancing the host defense against pancreatic inflammation. These studies enriched our knowledge of AP and laid the foundation for future translation work.

\section{Chronic pancreatitis}

$\mathrm{CP}$ with its damaged pancreatic acinar cells, can result in pancreatic exocrine insufficiency and small intestinal bacteria overgrowth. Small intestinal bacteria overgrowth appears to be more likely to occur in CP patients, due to intestinal dysmotility and reduced alkalization of intestinal fluid, as well as reduced pancreas-derived antimicrobial peptide. ${ }^{30}$ In a meta-analysis performed by Memba et al., ${ }^{31}$ three of 10 studies that met the inclusion criteria have assessed the gut flora in patients with $\mathrm{CP}^{32-34}$ They manifested that the abundance of Bifidobacterium or Lactobacillus were lower, while the abundance of Enterobacteriaceae was higher in CP patients (Table 1). Although data are still limited, lower levels of Bifidobacterium are observed both in AP and CP. Moreover, Bifidobacterium probably has beneficial role in other diseases like obesity, cystic fibrosis, inflammatory bowel disease, and irritable bowel syndrome. ${ }^{35}$ Clinically, this effect might be transformed into a potential therapeutic intervention to pancreatitis.

Autoimmune pancreatitis (AIP), a unique form of CP, 


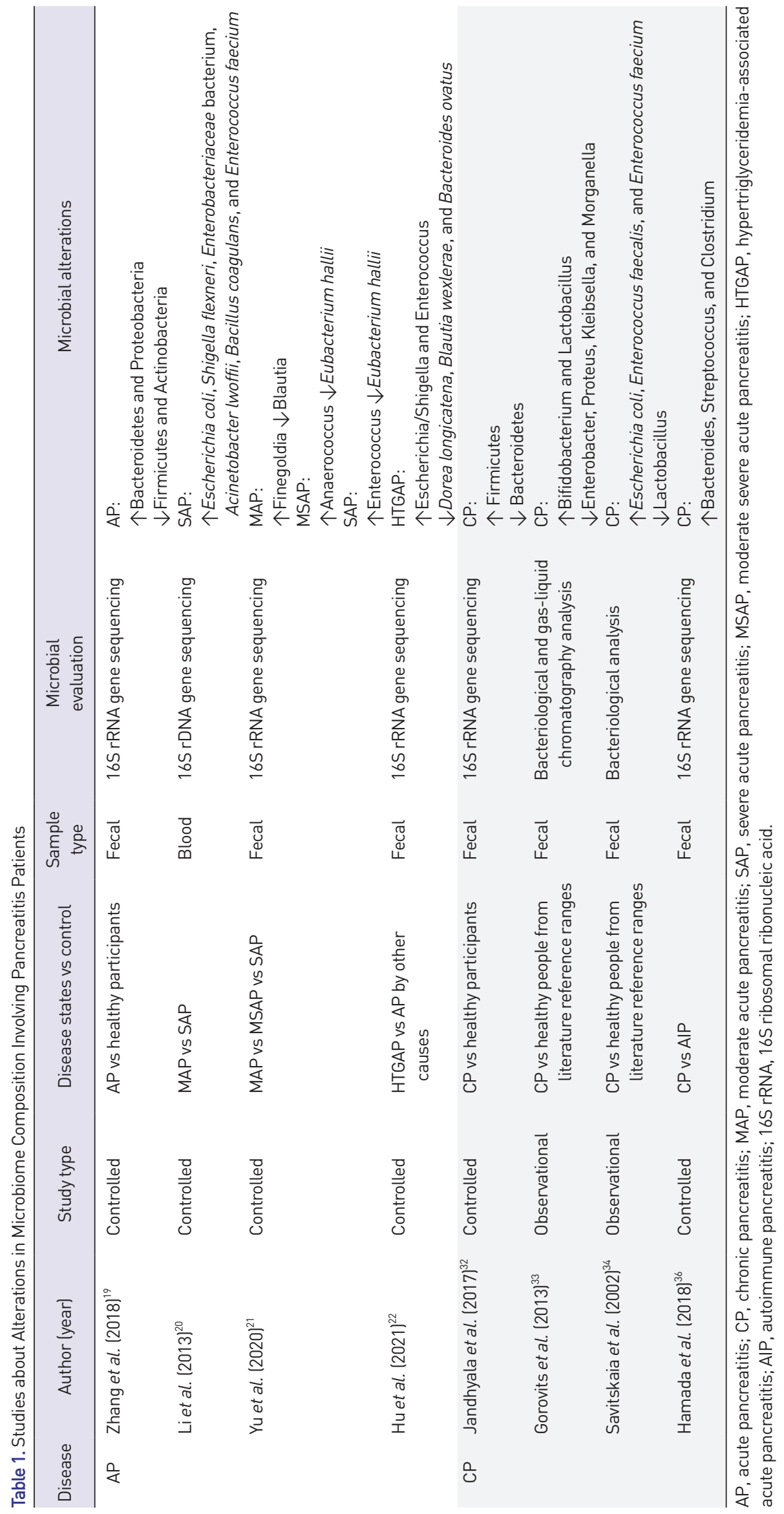


characterizes by storiform fibrosis and periductal lymphoplasmacytic infiltrate with or without granulocyte epithelial lesions, and depending on which to be divided into two types. ${ }^{37}$ The most important feature of AIP (solely for type 1 ) is the elevation of serum immunoglobulin G4. One study found that $E$. coli could generate AIP-like pathophysiological changes in the pancreas of control mice. ${ }^{38}$ Besides, this research also indicated that the antibody titers against $E$. coli in AIP patients were significantly higher than the titers of healthy controls. ${ }^{38}$ This may be another evidence that antigens of the intestinal microbiota could influence disease progression of pancreatic disorders. On the other hand, autoimmune response in AIP and malnutrition in CP could result in dysregulation of intestinal microbiota and influence gut microenvironment. Hamada et al. ${ }^{36}$ analyzed the fecal samples of eight CP patients and 12 AIP patients before steroids therapy. They found that no significant alterations were observed in gut microbiota between CP and AIP patients at the phylum level. ${ }^{36}$ However, Streptococcus, Bacteroides, and Clostridium were more abundant in the fecal samples of patients with CP compared with patients with AIP (Table 1). The reason for the elevated abundance of these bacterial species is still not very clear, but it may reflect a decrease in trypsin or malabsorption associated with CP.

\section{Pathogenesis hypothesis}

It is well-known that gut microbiota takes part in human physiological activities via influences on regulation of the mucosal immune system and intestinal architecture, involvement of digestion and metabolism. ${ }^{39}$ It is still controversial whether microorganisms inhabit normal pancreas. ${ }^{10,11}$ But it is a great chance that translocation of intestinal flora would occur, since the pancreas is linked to the gastrointestinal tract anatomically via the pancreatic duct and the route of mesenteric venous and lymphatic drainage (Fig. 1). Gut microbiota is confined to gastrointestinal tract in physiological conditions due to gastrointestinal mucosal barriers, including mechanical barrier, immune barrier, and biological barrier, which can effectively prevent the intestinal pathogenic bacteria and toxins to reach outside the gut. Once this homeostasis is disrupted, intestinal opportunistic pathogens can enter the blood circulation of AP patients through the damaged intestinal barrier, thus aggravating the progression of disease and the occurrence of infectious complications. In a meta-analysis of 18 studies, approximately $59 \%$ of patients with pancreatitis had intestinal barrier imbalance. ${ }^{40}$ Inflammation is the main pathophysiological response in pancreatitis, which is driven by either an infectious or a sterile event. Though bacteria are not the

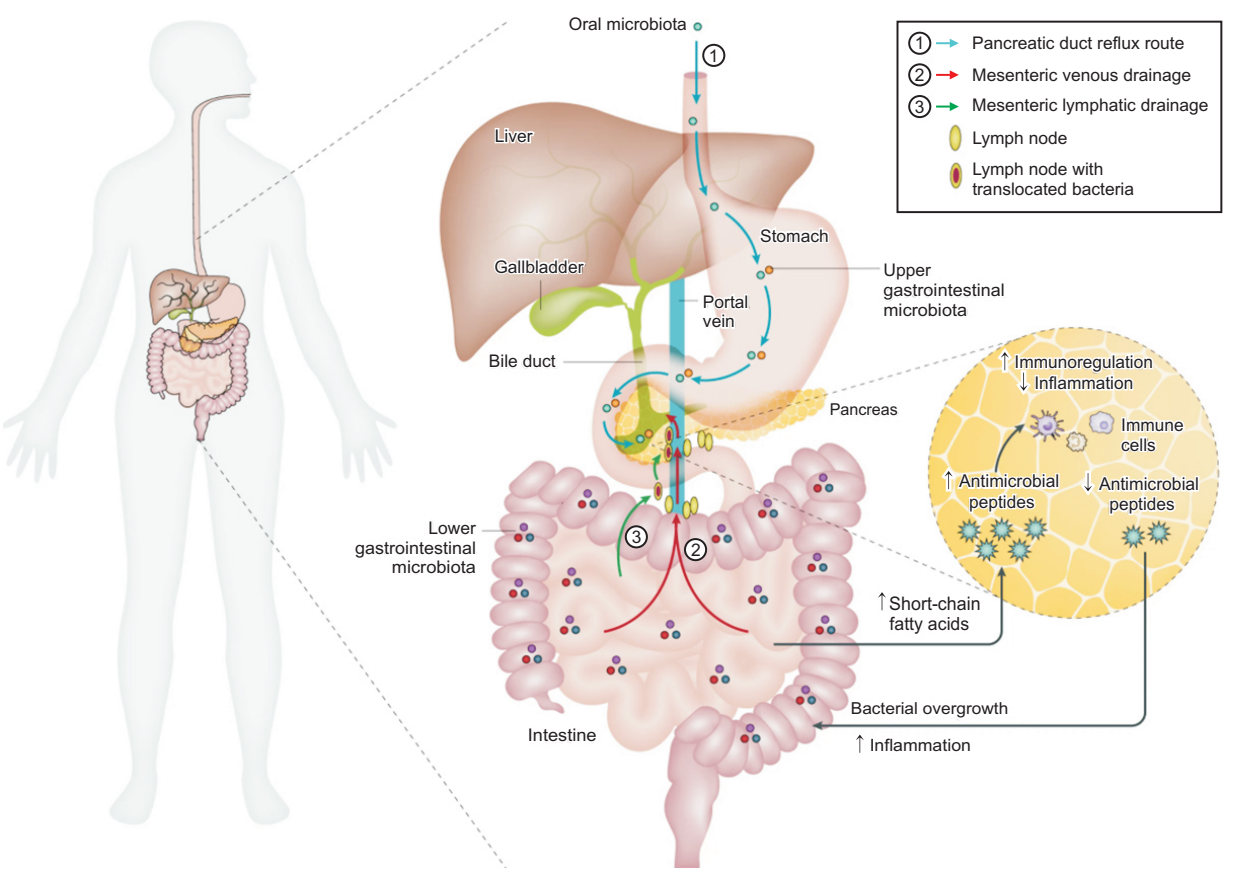

Fig. 1. Proposed routes of bacterial translocation to the pancreas and host response. The controversial routes whereby bacteria access the pancreas: however several mechanisms, such as the oral route (1), translocation from the lower gastrointestinal tract through the portal circulation (2), or mesenteric lymph nodes (3) are supported by the literature and are illustrated. Additionally, pancreatic antimicrobial peptides can have homeostatic bidirectional communication with the gastrointestinal tract, whereby the lower gastrointestinal microbiota influences pancreatic antimicrobial peptide production through short- chain fatty acid metabolites to induce an immunoregulatory pancreatic environment with decreased pro- inflammatory immune cells. Conversely, decreased antimicrobial peptide production by the pancreas enables gastrointestinal microbiota overgrowth and the development of a pro-inflammatory phenotype. Adapted from Thomas RM, et al. Nat Rev Gastroenterol Hepatol 2020;17:5364 , with permission from Springer Nature. ${ }^{41}$ 
directly cause of pancreatitis, the microorganisms can enter the pancreas in the inflammatory environment and aggravate the local and systemic inflammation. This is consistent with theories that the gut is the origin of clinical sepsis. ${ }^{42,43}$

In recent years, the role of immune cells in the pathogenesis of pancreatitis has been paid much attention, and further understanding of immune signaling pathway have been utilized to identify new therapeutic targets that may alter disease progression. ${ }^{44,45}$ The relationship between gut microbiota and host immune system is intimate and complex. Host-microbiota communication is mainly based on one group of host receptors, the pattern recognition receptors (PRRs) of the innate immune system, such as Toll-like receptors, C-type lectin receptors, and nucleotide-binding oligomerization domain (NOD)-like receptors. ${ }^{46-48}$ In pancreatic acinar cells, inflammation and immune response can be triggered by sensing of microorganism antigens by PRRs, which is involved in the pathogenesis of pancreatitis. ${ }^{39}$ In animal models, pancreatitis in mice model could be induced by chronic low-dose cerulein (cholecystokinin receptor agonist) stimulation collaborating with NOD1 agonist stimulation, while this effect is prevented in NOD1 knockout mice. ${ }^{49}$ One study indicated that gut microbiota could trigger non-infectious pancreatic inflammation through NOD1 signaling pathway in pancreatic acinar cells by binding to a peptide derived from peptidoglycan. ${ }^{50}$

Antimicrobial peptides (AMPs) are secretory components in the gastrointestinal tract. Though most proteins in pancreatic juice are contributed by digestive enzymes, the AMPs secreted by pancreatic acinar cells are also very important component of pancreatic juice. ${ }^{51,52}$ Pancreatic AMPs have a prominent role in regulating the gut microbiota that is essential for gut innate immunity. There is an intimate bidirectional communication between pancreatic AMPs and gut microbiota. On one hand, the lower gut flora could influence the production of pancreatic AMP to produce an immunoregulatory pancreatic environment by decreasing pro-inflammatory immune cells through shortchain fatty acids which are anti-inflammatory metabolites produced by intestinal microbiome, also facilitate integrity of intestinal epithelium. ${ }^{12}$ On the other hand, lack of AMP by the pancreas disrupts the gut microbiome homeostasis and leads to intestinal bacteria overgrowth and development of a pro-inflammatory status (Fig. 1). Additionally, the secretion of cathelicidin-related antimicrobial peptide (CRAMP) decreases when the $\mathrm{Ca}^{2+}$ channel Orail is knocked out in pancreatic acinar cells $\left(\mathrm{Orail}^{-{ }^{--}}\right.$) of adult mice, and resulted in systemic infection and high mortality rate due to intestinal bacterial overgrowth, elevated intestinal permeability and bacterial translocation. ${ }^{13}$

\section{THE ROLE OF GENETIC SUSCEPTIBILITY} OF PANCREATITIS

\section{Hereditary/familial pancreatitis}

Hereditary pancreatitis (HP) is defined as the condition in a family with two or more members suffered from recurrent acute pancreatitis (RAP) or CP in two or more generations, or perhaps pancreatitis which is associated with the pathogenic mutation of the cationic trypsinogen PRSS1 gene. ${ }^{53}$ This gain-of-function mutation of the cationic trypsinogen gene was first discovered by Whitcomb et al. in 1996, ${ }^{54}$ which brought new insights into pathogenesis of pancreatic disorders from the perspective of genetics. Most HP cases are inherited in autosomal dominant, whereas familial pancreatitis is used to describe recessive or complex phenotypes by clinical investigators or geneticists. ${ }^{55} \mathrm{HP}$ usually manifests as AP presented in childhood and subsequently resulting in the morphologic changes of $\mathrm{CP}$ with more frequent attack. As time going on, a variety of complications followed by $\mathrm{CP}$, including pancreatic fibrosis, pancreatic exocrine insufficiency, pancreatic ductal adenocarcinoma and so on, might emerge. ${ }^{56,57}$

Recent HP-related investigations have shown the mechanism and process of a primary susceptibility factor, such as PRSS1 R122H, turn into risk factors for AP and CP via $\mathrm{RAP}^{58-60}$ These findings confirmed the trypsin dependent theory in which gain-of-function mutations brought about trypsinogen or trypsin to be resistant to degradation. Besides, the activation of premature trypsin might take an alternate path resulting in RAP, thereafter part of the patients subsequently develop to $\mathrm{CP}$. There are some known genetic contributors to familial pancreatitis including lossof-function mutations of genes which encode the SPINK1, CFTR and variants in other genes. ${ }^{61-65}$ Whitcomb et al. ${ }^{66}$ have shown that the gene-environment interactions regarding HP are very complex by using a genome-wide association study analysis performed by next-generation sequencing.

\section{Genetic risk factors in pancreatitis}

The well-known mechanism of pancreatitis is trypsin premature activation, causing extensive zymogen activation, followed by pancreatic self-digestion, excessive immune response, and subsequent effects. ${ }^{67}$ Making use of candidate gene approaches, alterations in several distinct genes are associated with the regulation of trypsin in the pancreas, which is correlated with the pathogenesis of pancreatitis. To better understand the role of genetics in pancreatitis, we should firstly focus on the normal pancreas exocrine function, activity and regulation of trypsinogen, a zymogen precursor to trypsin. Trypsin is a protease produced and secreted by pancreatic acinar cells and upstream 
duct cells and activates other zymogens in the duodenum under a physiological state. Premature activation of trypsin could trigger an excessive, uncontrolled inflammatory response in pancreas, as seen in $\mathrm{AP}^{68}$ The two most common forms in pancreatic trypsinogen are the cationic (PRSS1) and anionic (PRSS2) forms. In the physiologic condition, autolysis could prevent from premature or excessive trypsin activation in pancreatic acinar and ductal cells. However pathogenic PRSS1 mutations can induce trypsin prematurely activated or degradation-resistant and meanwhile upgrades the level of autoactivation of mutant trypsinogens and trypsin activity within pancreas. ${ }^{8,63,69}$ As for PRSS2, pathogenic PRSS2 variants were not identified in HP or sporadic CP, whereas a variant in the noncoding region of the PRSS1-PRSS2 locus leads to a remarkably decrease in PRSS1 expression, mitigating the risk of pancreatitis. ${ }^{66,70}$ These mutations underline the importance of trypsinogen in the pathogenesis of pancreatitis.

During the inflammatory response of the pancreas, SPINK1 is significantly elevated to prevent excessive activation of trypsinogen and pancreatic damage through feedback inhibition of trypsin. This is the first line of defense against premature activation of intracellular trypsin. The most common p.N34S SPINK1 mutation was first mentioned to be correlated with $\mathrm{CP}$ in $2000 .^{71}$ Although the underlying mechanism of $\mathrm{CP}$ remains mystery, a meta-analysis has discovered that the SPINK1 N34S variant could increase the risk of alcoholic, idiopathic, and tropical $\mathrm{CP}^{72}$ Moreover, it seems to be essential for patients with heterozygous SPINK1 mutations to be linked to RAP or CP in collaborate with additional contributing factors related to recurrent activation of trypsin (like PRSS1 or CFTR) ${ }^{73-75}$ This suggests that heterozygous SPINK1 mutations could not increase susceptibility of pancreatitis directly, but aggravate recurrent pancreatic injury correlated to the activation of trypsin and promote the progression of $\mathrm{CP}$.

CFTR, an AMP-regulated anion channel located in epithelial cell membranes, mediates the secretion of bicarbonate-rich juice which is vital for secreting pancreatic zymogens. The dysfunction of the CFTR gene can make the acinar cells fail to alkalinize, resulting in zymogens remaining in the ducts, where they could become active and start to digest peripancreatic tissue, thus contributing to pancreatitis. CFTR mutations can affect the channel activity or membrane protein levels, and ultimately determine whether individuals would develop cystic fibrosis diseases and to what extent. While not only CFTR mutations causing cystic fibrosis are risk factors for pancreatitis, but those less penetrant CFTR alleles namely non-cystic fibrosiscausing variants, may also augment the risk of pancreatitis. Previous researches in different countries have indicated that individuals with idiopathic $\mathrm{CP}$ had higher rate of a CFTR mutation than the control group. ${ }^{76-78}$

Although the pathogenic role of PRSS1, SPINK1, and CFTR variants in pancreatitis is more widely known, a few uncommon genes also contribute to this process. These genes include calcium-sensing receptor (CASR), chymotrypsin C (CTRC), carboxypeptidase A1 (CPA1), and claudin-2 (CLDN2) gene, which are considered disease modifiers rather than disease initiators (Table 2, Fig. 2). ${ }^{79}$

\section{Genetic predisposition to alcoholic/HTG pancreatitis}

Although genetic etiology accounts for around 25\% of all cases of CP, it should be highlighted that about $40 \%$ of cases are thought to be idiopathic. ${ }^{80}$ The most common etiology of pancreatitis is still biliary disease, hypertriglyceridemia (HTG) and alcoholism. ${ }^{81}$ There have not been observed that genetic factors are involved in bile duct obstruction, pancreatic divisum, or the dysfunction of Oddi sphincter. The emergence of alcohol-related CP is often clustered in families, and this would further indicate a genetic predisposition. ${ }^{82}$ Epidemiological studies have unexpectedly found that only a small ratio of heavy drinkers (less than 3\%) would develop CP, but the risk of alcoholic pancreatitis is low when smoking is adjusted in regression analysis. ${ }^{7.83}$ Moreover, a threshold of more than five drinks a day ( 1 drink $=4 \mathrm{~g}$ of alcohol) or 35 drinks a week must be achieved before the risk of pancreatitis significantly increase. ${ }^{84}$ These observations suggest that alcohol consumption is stronger modifier factor than a susceptibility factor, especially with smoking ${ }^{83}$ and CLDN risk variants. ${ }^{66}$ The CLDN2 gene, encoding claudin-2, is expressed at low levels in pancreatic ducts as a tight junction protein. This high-risk gene variant triggers alcohol-related $\mathrm{CP}$ in men whose probability are greater compared with women with a high-risk locus near CLDN2 on the X chromosome correlated to pancreatitis. ${ }^{66,85,86}$ Further mechanisms of action of this risk locus need to be clarified.

Like alcohol-induced CP, only a small ratio of patients with HTG develop pancreatitis, which has inclined us to investigate genetic susceptibility factors. ${ }^{87}$ HTG-induced pancreatitis attacks typically from one or more secondary causes, such as medications, diabetes, alcoholism, pregnancy, in patients with potentially common genetic abnormalities of lipoprotein metabolism. Common variants in genes such as APOA5 (encoding apo A5), GCKR (encoding glucokinase regulatory protein), LPL (encoding lipoprotein lipase) and APOB (encoding apo B), associated with lipoprotein metabolism, can lead to a rise in serum triglyceridemia to the extent of incurring pancreatitis. ${ }^{88} \mathrm{~A}$ detailed process of triglyceridemia metabolism can refer 


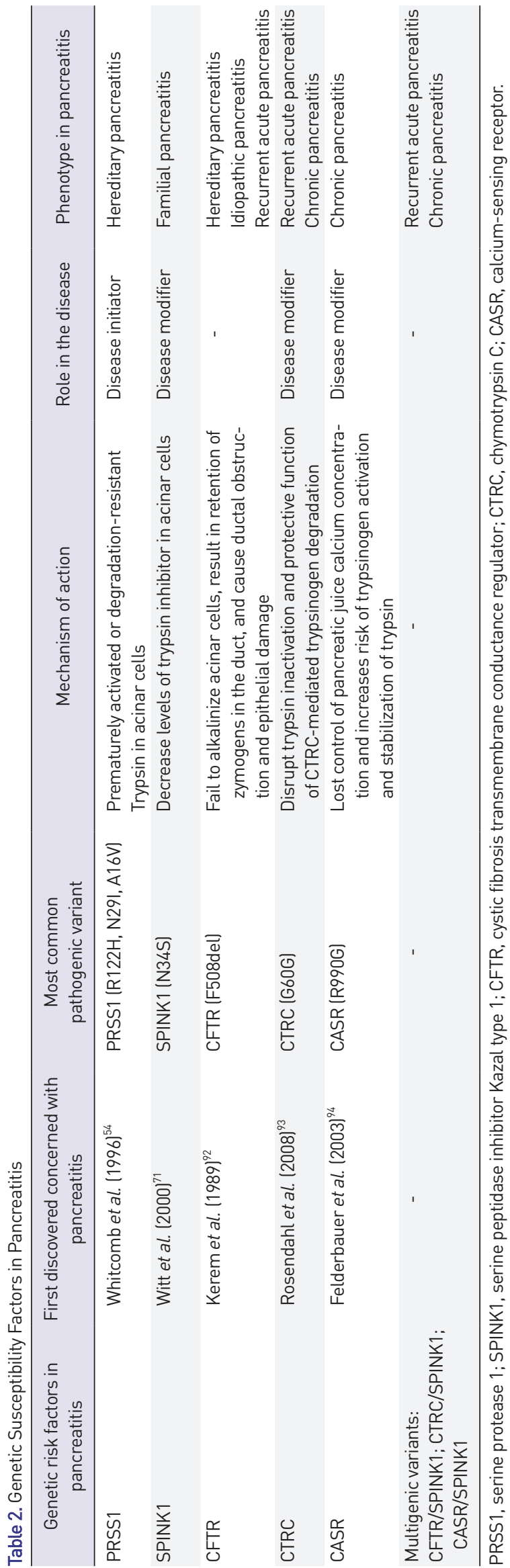

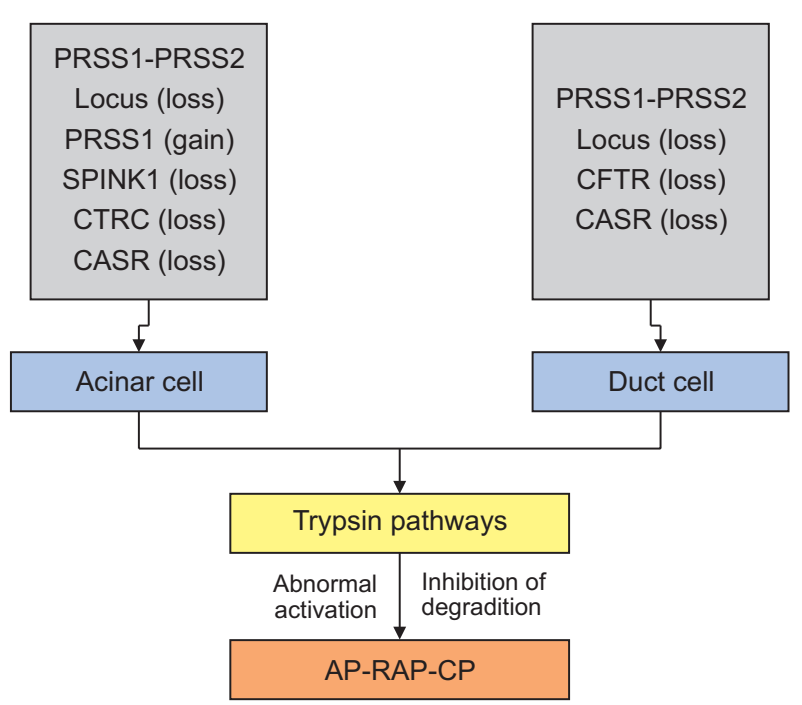

Fig. 2. Genetic susceptibility factors related to the trypsin pathways to pancreatitis. A summary of genetic factors that contribute to the pathogenesis of pancreatitis.

$A P$, acute pancreatitis; RAP, recurrent acute pancreatitis; $C P$, chronic pancreatitis; PRSS1, serine protease 1; PRSS2, serine protease 2; SPINK1, serine peptidase inhibitor Kazal type 1; CFTR, cystic fibrosis transmembrane conductance regulator; CTRC, chymotrypsin C; CASR, calcium-sensing receptor.

to elsewhere (see review) ${ }^{89}$ Focused on genetic factors to HTG pancreatitis, Chang et al. ${ }^{90}$ assessed the frequency of mutations in PRSS1, SPINK1, CFTR, and tumor necrosis factor superfamily member 2 (TNF2) genes in 126 HTG patients including 46 patients with hyperlipidemic pancreatitis (HLP) and 80 patients without HLP. The frequency of CFTR (M470V) and TNF (863A) mutations in HLP patients was significantly higher than patients with HTG alone, which showed that CFTR mutation and TNF promoter polymorphism probably involved in the development of HLP in HTG patients. In another study, a cohort of patients with severe, intractable HTG (triglyceride level above 2,000 mg/dL) with and without AP, AP group were significantly younger with higher fasting glucose and lower high-density lipoprotein cholesterol, indicating a stronger genetic background for HTG in this group. ${ }^{91}$ More researches are necessary to investigate the role of genetic factors in increasing the risk of pancreatitis in patients suffered from severe/critically severe HTG.

\section{ASSOCIATION BETWEEN GUT MICROBIOTA AND GENETIC VARIATIONS IN THE PATHOGENESIS OF PANCREATITIS}

The causation between microbiota and host genetics remains to be elucidated, since our knowledge of the host side is limited and recognition of which bacterial genes are 
implementing the crosstalk with the host is poorer. ${ }^{95}$ What we have already known is that the gut microbiota diversity, structure, and composition are associated with host genetic variations. ${ }^{9697}$ These associations are specifically motivated by host genetic variation in immunity-related pathways. ${ }^{98}$ Meanwhile, one report demonstrates that genetic risk for developing type 1 diabetes autoimmunity is linked with significant changes in the gut microbiota, ${ }^{99}$ which is a manifestation of interaction between gut microbiota and host genetic factors in pancreas disorders. As for pancreatitis, another study reported that children with CP who carry different genetic variations concerned with abnormal activation of trypsinogen and secretions in the pancreatic duct present different abundances of gut microbiota genera. ${ }^{100}$ Their findings support that disordered gut microbiota may affect host gene expression and then disturbing normal physiology function and contributing to the development of disease. On basis of above evidence, we can raise the hypothesis that the pathogenesis of pancreatitis might be influenced by the interactions of both genetic and microbial factors. However, the in-depth mechanism needs to be further investigated.

\section{CONCLUSIONS}

Growing evidence regarding the role of gut microbiota and genetic variations in pathophysiologic mechanism of pancreatitis has provided us with new insights into AP and CP. We now know that pancreatitis is not only a dysfunction of acinar cells, but a multi-factorial complicated pancreatic disorder involving gut microbiota, host immune system, environmental factors, and genetic causes. Although mechanistic understanding of these two rare factors is limited, it is clear that continued advances in bacteria-related function and genomic technologies would act as novel therapeutic interventions for pancreatitis in the near future.

\section{CONFLICTS OF INTEREST}

No potential conflict of interest relevant to this article was reported.

\section{ACKNOWLEDGEMENTS}

This work was supported by the National Natural Science Foundation of China (number: 81700483), Chongqing Research Program of Basic Research and Frontier
Technology (numbers: cstc2017jcyjAX0302, cstc2020jcyjmsxmX1100), Frontier Technology Research Program of Army Medical University (number: 2019XLC3051).

\section{ORCID}

Fumin Xu https://orcid.org/0000-0002-3042-8054 Chunmei Yang https://orcid.org/0000-0002-0743-6873 Mingcheng Tang https://orcid.org/0000-0002-1622-1591 Ming Wang https://orcid.org/0000-0002-3687-6384 Zhenhao Cheng https://orcid.org/0000-0003-0752-693X Dongfeng Chen https://orcid.org/0000-0003-1700-2195 Xiao Chen https://orcid.org/0000-0002-8647-0614 Kaijun Liu https://orcid.org/0000-0003-0027-9278

\section{REFERENCES}

1. Peery AF, Crockett SD, Murphy CC, et al. Burden and cost of gastrointestinal, liver, and pancreatic diseases in the United States: update 2018. Gastroenterology 2019;156:254-272.

2. Petrov MS, Yadav D. Global epidemiology and holistic prevention of pancreatitis. Nat Rev Gastroenterol Hepatol 2019;16:175-184.

3. Lugea A, Waldron RT, Mareninova OA, et al. Human pancreatic acinar cells: proteomic characterization, physiologic responses, and organellar disorders in ex vivo pancreatitis. Am J Pathol 2017;187:2726-2743.

4. Gukovskaya AS, Pandol SJ, Gukovsky I. New insights into the pathways initiating and driving pancreatitis. Curr Opin Gastroenterol 2016;32:429-435.

5. Banks PA, Bollen TL, Dervenis C, et al. Classification of acute pancreatitis: 2012. Revision of the Atlanta classification and definitions by international consensus. Gut 2013;62:102111.

6. Lee PJ, Papachristou GI. New insights into acute pancreatitis. Nat Rev Gastroenterol Hepatol 2019;16:479-496.

7. Yadav D, Eigenbrodt ML, Briggs MJ, Williams DK, Wiseman EJ. Pancreatitis: prevalence and risk factors among male veterans in a detoxification program. Pancreas 2007;34:390398.

8. Whitcomb DC. Genetic risk factors for pancreatic disorders. Gastroenterology 2013;144:1292-1302.

9. Brestoff JR, Artis D. Commensal bacteria at the interface of host metabolism and the immune system. Nat Immunol 2013;14:676-684.

10. Thomas RM, Gharaibeh RZ, Gauthier J, et al. Intestinal microbiota enhances pancreatic carcinogenesis in preclinical models. Carcinogenesis 2018;39:1068-1078.

11. Pushalkar S, Hundeyin M, Daley D, et al. The pancreatic 
cancer microbiome promotes oncogenesis by induction of innate and adaptive immune suppression. Cancer Discov 2018;8:403-416.

12. Sun J, Furio L, Mecheri R, et al. Pancreatic $\beta$-cells limit autoimmune diabetes via an immunoregulatory antimicrobial peptide expressed under the influence of the gut microbiota. Immunity 2015;43:304-317.

13. Ahuja M, Schwartz DM, Tandon M, et al. Orail-mediated antimicrobial secretion from pancreatic acini shapes the gut microbiome and regulates gut innate immunity. Cell Metab 2017;25:635-646.

14. Geller LT, Barzily-Rokni M, Danino T, et al. Potential role of intratumor bacteria in mediating tumor resistance to the chemotherapeutic drug gemcitabine. Science 2017;357:11561160.

15. Aghdassi AA, Weiss FU, Mayerle J, Lerch MM, Simon P. Genetic susceptibility factors for alcohol-induced chronic pancreatitis. Pancreatology 2015;15(4 Suppl):S23-S31.

16. Solomon S, Whitcomb DC. Genetics of pancreatitis: an update for clinicians and genetic counselors. Curr Gastroenterol Rep 2012;14:112-117.

17. Chandak GR, Idris MM, Reddy DN, et al. Absence of PRSS1 mutations and association of SPINK1 trypsin inhibitor mutations in hereditary and non-hereditary chronic pancreatitis. Gut 2004;53:723-728.

18. Lerch MM, Gorelick FS. Models of acute and chronic pancreatitis. Gastroenterology 2013;144:1180-1193.

19. Zhang XM, Zhang ZY, Zhang CH, Wu J, Wang YX, Zhang GX. Intestinal microbial community differs between acute pancreatitis patients and healthy volunteers. Biomed Environ Sci 2018;31:81-86.

20. Li Q, Wang C, Tang C, He Q, Li N, Li J. Bacteremia in patients with acute pancreatitis as revealed by $16 \mathrm{~S}$ ribosomal RNA gene-based techniques*. Crit Care Med 2013;41:19381950.

21. Yu S, Xiong Y, Xu J, et al. Identification of dysfunctional gut microbiota through rectal swab in patients with different severity of acute pancreatitis. Dig Dis Sci 2020;65:3223-3237.

22. $\mathrm{Hu} \mathrm{X}$, Gong L, Zhou R, et al. Variations in gut microbiome are associated with prognosis of hypertriglyceridemia-associated acute pancreatitis. Biomolecules 2021;11:695.

23. Zhu Y, He C, Li X, et al. Gut microbiota dysbiosis worsens the severity of acute pancreatitis in patients and mice. J Gastroenterol 2019;54:347-358.

24. Besselink MG, van Santvoort HC, Buskens E, et al. Probiotic prophylaxis in predicted severe acute pancreatitis: a randomised, double-blind, placebo-controlled trial. Lancet 2008;371:651-659.

25. Oláh A, Belágyi T, PótóL, Romics L Jr, Bengmark S. Synbiotic control of inflammation and infection in severe acute pancreatitis: a prospective, randomized, double blind study.
Hepatogastroenterology 2007;54:590-594

26. Cui LH, Wang XH, Peng LH, Yu L, Yang YS. The effects of early enteral nutrition with addition of probiotics on the prognosis of patients suffering from severe acute pancreatitis. Zhonghua Wei Zhong Bing Ji Jiu Yi Xue 2013;25:224228.

27. Plaudis H, Pupelis G, Zeiza K, Boka V. Early low volume oral synbiotic/prebiotic supplemented enteral stimulation of the gut in patients with severe acute pancreatitis: a prospective feasibility study. Acta Chir Belg 2012;112:131-138.

28. Yu C, Zhang Y, Yang Q, Lee P, Windsor JA, Wu D. An updated systematic review with meta-analysis: efficacy of prebiotic, probiotic, and synbiotic treatment of patients with severe acute pancreatitis. Pancreas 2021;50:160-166.

29. Lei Y, Tang L, Liu S, et al. Parabacteroides produces acetate to alleviate heparanase-exacerbated acute pancreatitis through reducing neutrophil infiltration. Microbiome 2021;9:115.

30. Bures J, Cyrany J, Kohoutova D, et al. Small intestinal bacterial overgrowth syndrome. World J Gastroenterol 2010;16:2978-2990.

31. Memba R, Duggan SN, Ni Chonchubhair HM, et al. The potential role of gut microbiota in pancreatic disease: a systematic review. Pancreatology 2017;17:867-874.

32. Jandhyala SM, Madhulika A, Deepika G, et al. Altered intestinal microbiota in patients with chronic pancreatitis: implications in diabetes and metabolic abnormalities. Sci Rep 2017;7:43640.

33. Gorovits ES, Tokareva EV, Khlynova OV, Zhelobov VG, El'kin VD. Complex evaluation of intestine microbiocenosis condition in patients with chronic pancreatitis. Zh Mikrobiol Epidemiol Immunobiol 2013;(4):73-76.

34. Savitskaia KI, Mel'nikova EF, Vorob'ev AA, Zagal'skaia NV. Evaluation of microecology of colonic contents in patients with chronic pancreatitis. Vestn Ross Akad Med Nauk 2002;(4):20-23.

35. Leal-Lopes C, Velloso FJ, Campopiano JC, Sogayar MC, Correa RG. Roles of commensal microbiota in pancreas homeostasis and pancreatic pathologies. J Diabetes Res 2015;2015:284680.

36. Hamada S, Masamune A, Nabeshima T, Shimosegawa T. Differences in gut microbiota profiles between autoimmune pancreatitis and chronic pancreatitis. Tohoku J Exp Med 2018;244:113-117.

37. Shimosegawa T, Chari ST, Frulloni L, et al. International consensus diagnostic criteria for autoimmune pancreatitis: guidelines of the International Association of Pancreatology. Pancreas 2011;40:352-358.

38. Yanagisawa N, Haruta I, Shimizu K, et al. Identification of commensal flora-associated antigen as a pathogenetic factor of autoimmune pancreatitis. Pancreatology 2014;14:100-106.

39. Akshintala VS, Talukdar R, Singh VK, Goggins M. The gut 
microbiome in pancreatic disease. Clin Gastroenterol Hepatol 2019;17:290-295.

40. Wu LM, Sankaran SJ, Plank LD, Windsor JA, Petrov MS. Meta-analysis of gut barrier dysfunction in patients with acute pancreatitis. Br J Surg 2014;101:1644-1656.

41. Thomas RM, Jobin C. Microbiota in pancreatic health and disease: the next frontier in microbiome research. Nat Rev Gastroenterol Hepatol 2020;17:53-64.

42. O'Boyle CJ, MacFie J, Mitchell CJ, Johnstone D, Sagar PM, Sedman PC. Microbiology of bacterial translocation in humans. Gut 1998;42:29-35.

43. MacFie J, O'Boyle C, Mitchell CJ, Buckley PM, Johnstone D, Sudworth P. Gut origin of sepsis: a prospective study investigating associations between bacterial translocation, gastric microflora, and septic morbidity. Gut 1999;45:223-228.

44. Sendler M, Weiss FU, Golchert J, et al. Cathepsin B-mediated activation of trypsinogen in endocytosing macrophages increases severity of pancreatitis in mice. Gastroenterology 2018;154:704-718.

45. Antonucci L, Fagman JB, Kim JY, et al. Basal autophagy maintains pancreatic acinar cell homeostasis and protein synthesis and prevents ER stress. Proc Natl Acad Sci U S A 2015;112:E6166-E6174.

46. Chu H, Mazmanian SK. Innate immune recognition of the microbiota promotes host-microbial symbiosis. Nat Immunol 2013;14:668-675.

47. Brown RL, Clarke TB. The regulation of host defences to infection by the microbiota. Immunology 2017;150:1-6.

48. Rosenstiel P, Philipp EE, Schreiber S, Bosch TC. Evolution and function of innate immune receptors: insights from marine invertebrates. J Innate Immun 2009;1:291-300.

49. Watanabe T, Sadakane Y, Yagama N, et al. Nucleotidebinding oligomerization domain 1 acts in concert with the cholecystokinin receptor agonist, cerulein, to induce IL-33-dependent chronic pancreatitis. Mucosal Immunol 2016;9:1234-1249.

50. Tsuji Y, Watanabe T, Kudo M, Arai H, Strober W, Chiba T. Sensing of commensal organisms by the intracellular sensor NOD1 mediates experimental pancreatitis. Immunity 2012;37:326-338.

51. Medveczky P, Szmola R, Sahin-Tóth M. Proteolytic activation of human pancreatitis-associated protein is required for peptidoglycan binding and bacterial aggregation. Biochem J 2009;420:335-343.

52. Doyle CJ, Yancey K, Pitt HA, et al. The proteome of normal pancreatic juice. Pancreas 2012;41:186-194.

53. Shelton C, LaRusch J, Whitcomb DC. Pancreatitis overview. In: Adam MP, Ardinger HH, Pagon RA, Wallace SE, eds. GeneReviews ${ }^{\oplus}$ [Internet]. Seattle: University of Washington, 1993-2021. Available from: https://www.ncbi.nlm.nih.gov/ books/NBK190101/.
54. Whitcomb DC, Gorry MC, Preston RA, et al. Hereditary pancreatitis is caused by a mutation in the cationic trypsinogen gene. Nat Genet 1996;14:141-145.

55. Bombieri C, Claustres M, De Boeck K, et al. Recommendations for the classification of diseases as CFTR-related disorders. J Cyst Fibros 2011;10 Suppl 2:S86-S102.

56. Amann ST, Gates LK, Aston CE, Pandya A, Whitcomb DC. Expression and penetrance of the hereditary pancreatitis phenotype in monozygotic twins. Gut 2001;48:542-547.

57. Joergensen MT, Brusgaard K, Crüger DG, Gerdes AM, Schaffalitzky de Muckadell OB. Genetic, epidemiological, and clinical aspects of hereditary pancreatitis: a populationbased cohort study in Denmark. Am J Gastroenterol 2010;105:1876-1883.

58. Gorry MC, Gabbaizedeh D, Furey W, et al. Mutations in the cationic trypsinogen gene are associated with recurrent acute and chronic pancreatitis. Gastroenterology 1997;113:10631068 .

59. Howes N, Lerch MM, Greenhalf W, et al. Clinical and genetic characteristics of hereditary pancreatitis in Europe. Clin Gastroenterol Hepatol 2004;2:252-261.

60. Rebours V, Boutron-Ruault MC, Schnee M, et al. The natural history of hereditary pancreatitis: a national series. Gut 2009;58:97-103.

61. Witt H, Apte MV, Keim V, Wilson JS. Chronic pancreatitis: challenges and advances in pathogenesis, genetics, diagnosis, and therapy. Gastroenterology 2007;132:1557-1573.

62. Chen JM, Férec C. Chronic pancreatitis: genetics and pathogenesis. Annu Rev Genomics Hum Genet 2009;10:63-87.

63. Whitcomb DC. Genetic aspects of pancreatitis. Annu Rev Med 2010;61:413-424.

64. LaRusch J, Whitcomb DC. Genetics of pancreatitis. Curr Opin Gastroenterol 2011;27:467-474.

65. Chen JM, Férec C. Genetics and pathogenesis of chronic pancreatitis: the 2012 update. Clin Res Hepatol Gastroenterol 2012;36:334-340.

66. Whitcomb DC, LaRusch J, Krasinskas AM, et al. Common genetic variants in the CLDN2 and PRSS1-PRSS2 loci alter risk for alcohol-related and sporadic pancreatitis. Nat Genet 2012;44:1349-1354.

67. Whitcomb DC. Mechanisms of disease: advances in understanding the mechanisms leading to chronic pancreatitis. Nat Clin Pract Gastroenterol Hepatol 2004;1:46-52.

68. Zator Z, Whitcomb DC. Insights into the genetic risk factors for the development of pancreatic disease. Therap Adv Gastroenterol 2017;10:323-336.

69. Hegyi E, Sahin-Tóth M. Genetic risk in chronic pancreatitis: the trypsin-dependent pathway. Dig Dis Sci 2017;62:16921701.

70. Witt H, Sahin-Tóth M, Landt O, et al. A degradation-sensitive anionic trypsinogen (PRSS2) variant protects against 
chronic pancreatitis. Nat Genet 2006;38:668-673.

71. Witt H, Luck W, Hennies HC, et al. Mutations in the gene encoding the serine protease inhibitor, Kazal type 1 are associated with chronic pancreatitis. Nat Genet 2000;25:213-216.

72. Aoun E, Chang CC, Greer JB, Papachristou GI, Barmada MM, Whitcomb DC. Pathways to injury in chronic pancreatitis: decoding the role of the high-risk SPINK1 N34S haplotype using meta-analysis. PLoS One 2008;3:e2003.

73. Midha S, Khajuria R, Shastri S, Kabra M, Garg PK. Idiopathic chronic pancreatitis in India: phenotypic characterisation and strong genetic susceptibility due to SPINK1 and CFTR gene mutations. Gut 2010;59:800-807.

74. Rosendahl J, Landt O, Bernadova J, et al. CFTR, SPINK1, CTRC and PRSS1 variants in chronic pancreatitis: is the role of mutated CFTR overestimated? Gut 2013;62:582-592.

75. Schneider A, Larusch J, Sun X, et al. Combined bicarbonate conductance-impairing variants in CFTR and SPINK1 variants are associated with chronic pancreatitis in patients without cystic fibrosis. Gastroenterology 2011;140:162-171.

76. Weiss FU, Simon P, Bogdanova N, et al. Complete cystic fibrosis transmembrane conductance regulator gene sequencing in patients with idiopathic chronic pancreatitis and controls. Gut 2005;54:1456-1460.

77. Choudari CP, Imperiale TF, Sherman S, Fogel E, Lehman GA. Risk of pancreatitis with mutation of the cystic fibrosis gene. Am J Gastroenterol 2004;99:1358-1363.

78. Castellani C, Bonizzato A, Rolfini R, Frulloni L, Cavallini GC, Mastella G. Increased prevalence of mutations of the cystic fibrosis gene in idiopathic chronic and recurrent pancreatitis. Am J Gastroenterol 1999;94:1993-1995.

79. Hasan A, Moscoso DI, Kastrinos F. The role of genetics in pancreatitis. Gastrointest Endosc Clin N Am 2018;28:587-603.

80. Keller J, Layer P. Idiopathic chronic pancreatitis. Best Pract Res Clin Gastroenterol 2008;22:105-113.

81. Kleeff J, Whitcomb DC, Shimosegawa T, et al. Chronic pancreatitis. Nat Rev Dis Primers 2017;3:17060.

82. Schneider A, Pfützer RH, Barmada MM, Slivka A, Martin J, Whitcomb DC. Limited contribution of the SPINK1 N34S mutation to the risk and severity of alcoholic chronic pancreatitis: a report from the United States. Dig Dis Sci 2003;48:1110-1115

83. Yadav D, Hawes RH, Brand RE, et al. Alcohol consumption, cigarette smoking, and the risk of recurrent acute and chronic pancreatitis. Arch Intern Med 2009;169:1035-1045.

84. Yadav D, Whitcomb DC. The role of alcohol and smoking in pancreatitis. Nat Rev Gastroenterol Hepatol 2010;7:131-145.

85. Derikx MH, Kovacs P, Scholz M, et al. Polymorphisms at PRSS1-PRSS2 and CLDN2-MORC4 loci associate with alcoholic and non-alcoholic chronic pancreatitis in a European replication study. Gut 2015;64:1426-1433.

86. Masamune A, Nakano E, Hamada S, Kakuta Y, Kume K,
Shimosegawa T. Common variants at PRSS1-PRSS2 and CLDN2-MORC4 loci associate with chronic pancreatitis in Japan. Gut 2015;64:1345-1346.

87. Scherer J, Singh VP, Pitchumoni CS, Yadav D. Issues in hypertriglyceridemic pancreatitis: an update. J Clin Gastroenterol 2014;48:195-203.

88. Johansen CT, Hegele RA. Genetic bases of hypertriglyceridemic phenotypes. Curr Opin Lipidol 2011;22:247-253.

89. Alves-Bezerra M, Cohen DE. Triglyceride metabolism in the liver. Compr Physiol 2017;8:1-8.

90. Chang YT, Chang MC, Su TC, et al. Association of cystic fibrosis transmembrane conductance regulator (CFTR) mutation/variant/haplotype and tumor necrosis factor (TNF) promoter polymorphism in hyperlipidemic pancreatitis. Clin Chem 2008;54:131-138.

91. Juliani FC, Miname MH, Chacra APM, et al. Predisposing factors to acute pancreatitis in patients with severe hypertriglyceridemia. Eur Heart J 2020;41(Suppl 2):ehaa946.2984.

92. Kerem B, Rommens JM, Buchanan JA, et al. Identification of the cystic fibrosis gene: genetic analysis. Science 1989;245:1073-1080.

93. Rosendahl J, Witt H, Szmola R, et al. Chymotrypsin C (CTRC) variants that diminish activity or secretion are associated with chronic pancreatitis. Nat Genet 2008;40:78-82.

94. Felderbauer P, Hoffmann P, Einwächter H, et al. A novel mutation of the calcium sensing receptor gene is associated with chronic pancreatitis in a family with heterozygous SPINK1 mutations. BMC Gastroenterol 2003;3:34.

95. Wang J, Chen L, Zhao N, Xu X, Xu Y, Zhu B. Of genes and microbes: solving the intricacies in host genomes. Protein Cell 2018;9:446-461.

96. Rothhammer V, Mascanfroni ID, Bunse L, et al. Type I interferons and microbial metabolites of tryptophan modulate astrocyte activity and central nervous system inflammation via the aryl hydrocarbon receptor. Nat Med 2016;22:586597.

97. Jones EA, Kananurak A, Bevins CL, Hollox EJ, Bakaletz LO. Copy number variation of the beta defensin gene cluster on chromosome $8 \mathrm{p}$ influences the bacterial microbiota within the nasopharynx of otitis-prone children. PLoS One 2014;9:e98269.

98. Blekhman R, Goodrich JK, Huang K, et al. Host genetic variation impacts microbiome composition across human body sites. Genome Biol 2015;16:191.

99. Russell JT, Roesch LFW, Ördberg M, et al. Genetic risk for autoimmunity is associated with distinct changes in the human gut microbiome. Nat Commun 2019;10:3621.

100. Wang W, Xiao Y, Wang X, et al. Disordered gut microbiota in children who have chronic pancreatitis and different functional gene mutations. Clin Transl Gastroenterol 2020;11:e00150. 